\title{
Existence and Ulam stability for fractional differential equations of Hilfer-Hadamard type
}

\author{
S Abbas ${ }^{1}$, M Benchohra ${ }^{2}$, JE Lagreg ${ }^{2}$, A Alsaedi $^{3}$ and $Y$ Zhou $^{4,3 *}$
}

\section{"Correspondence:}

yzhou@xtu.edu.cn

${ }^{3}$ Nonlinear Analysis and Applied

Mathematics (NAAM) Research

Group, Faculty of Science, King

Abdulaziz University, P.O. Box 80203,

Jeddah, 21589, Saudi Arabia

${ }^{4}$ Faculty of Mathematics and

Computational Science, Xiangtan

University, Hunan, 411105, P.R. China

Full list of author information is

available at the end of the article

\begin{abstract}
This article deals with some existence and Ulam-Hyers-Rassias stability results for a class of functional differential equations involving the Hilfer-Hadamard fractional derivative. An application is made of a Schauder fixed point theorem for the existence of solutions. Next we prove that our problem is generalized Ulam-Hyers-Rassias stable.
\end{abstract}

MSC: 26A33; 34A08; 34K37

Keywords: functional differential equation; left-sided mixed Hadamard fractional integral; Hilfer-Hadamard fractional derivative; existence; Ulam-Hyers-Rassias stability

\section{Introduction}

Fractional differential equations have recently been applied in various areas of engineering, mathematics, physics and bio-engineering, and other applied sciences. For some fundamental results in the theory of fractional calculus and fractional ordinary and partial differential equations, we refer the reader to the monographs of Abbas et al. [1, 2], Samko et al. [3], Kilbas et al. [4] and Zhou [5], the papers [6-22] and the references therein.

The stability of functional equations was originally raised by Ulam [23], next by Hyers [24]. Thereafter, this type of stability is called the Ulam-Hyers stability. In 1978, Rassias [25] provided a remarkable generalization of the Ulam-Hyers stability of mappings by considering variables. The concept of stability for a functional equation arises when we replace the functional equation by an inequality which acts as a perturbation of the equation. Considerable attention has been given to the study of the Ulam-Hyers and Ulam-HyersRassias stability of all kinds of functional equations; one can see the monographs of [26], and the papers of Abbas et al. [6, 8, 9, 27-29], Petru et al. [30], Rus [31, 32], and Wang et $a l$. [33, 34]. More details from historical point of view, and recent developments of such stabilities are reported in $[31,35]$.

Recently, considerable attention has been given to the existence of solutions of initial and boundary value problems for fractional differential equations with Hilfer fractional derivative; see [36-42]. Motivated by the Hilfer fractional derivative (which interpolates the Riemann-Liouville derivative and the Caputo derivative), Qassim et al. [43, 44] considered a new type of fractional derivative (which interpolates the Hadamard derivative and its Caputo counterpart). Motivated by the above papers, in this article we discuss the

(c) The Author(s) 2017. This article is distributed under the terms of the Creative Commons Attribution 4.0 International License (http://creativecommons.org/licenses/by/4.0/), which permits unrestricted use, distribution, and reproduction in any medium, provided you give appropriate credit to the original author(s) and the source, provide a link to the Creative Commons license, and indicate if changes were made. 
existence and the Ulam stability of solutions for the following problem of Hilfer-Hadamard fractional differential equations of the form

$$
\left\{\begin{array}{l}
\left({ }^{H} D_{1}^{\alpha, \beta} u\right)(t)=f(t, u(t)) ; \quad t \in J:=[1, T] \\
\left.\left({ }^{H} I_{1}^{1-\gamma} u\right)(t)\right|_{t=1}=\phi
\end{array}\right.
$$

where $\alpha \in(0,1), \beta \in[0,1], \gamma=\alpha+\beta-\alpha \beta, T>1, \phi \in \mathbb{R}, f: J \times \mathbb{R} \rightarrow \mathbb{R}$ is a given function, ${ }^{H} I_{1}^{1-\gamma}$ is the left-sided mixed Hadamard integral of order $1-\gamma$, and ${ }^{H} D_{1}^{\alpha, \beta}$ is the HilferHadamard fractional derivative of order $\alpha$ and type $\beta$, introduced by Hilfer in [38].

The present paper initiates the Ulam stability for differential equations involving the Hilfer-Hadamard fractional derivative.

\section{Preliminaries}

Let $C$ be the Banach space of all continuous functions $v$ from $I$ into $\mathbb{R}$ with the supremum (uniform) norm

$$
\|v\|_{\infty}:=\sup _{t \in J}|v(t)|
$$

By $L^{1}(J)$, we denote the space of Lebesgue-integrable functions $v: J \rightarrow \mathbb{R}$ with the norm

$$
\|v\|_{1}=\int_{0}^{T}|v(t)| d t
$$

As usual, $\mathrm{AC}(J)$ denotes the space of absolutely continuous functions from $J$ into $\mathbb{R}$. We denote by $\operatorname{AC}^{1}(J)$ the space defined by

$$
\operatorname{AC}^{1}(J):=\left\{w: J \rightarrow \mathbb{R}: \frac{d}{d t} w(t) \in \operatorname{AC}(J)\right\}
$$

Let

$$
\delta=t \frac{d}{d t}, \quad q>0, \quad n=[q]+1,
$$

where $[q]$ is the integer part of $q$. Define the space

$$
\mathrm{AC}_{\delta}^{n}:=\left\{u:[1, T] \rightarrow E: \delta^{n-1}[u(t)] \in \mathrm{AC}(J)\right\}
$$

Let $\gamma \in(0,1]$, by $C_{\gamma, \ln }(J), C_{\gamma}(J)$ and $C_{\gamma}^{1}(J)$, we denote the weighted spaces of continuous functions defined by

$$
C_{\gamma, \ln }(J)=\left\{w(t):(\ln t)^{1-\gamma} w(t) \in C\right\}
$$

with the norm

$$
\begin{aligned}
& \|w\|_{C_{\gamma, \mathrm{ln}}}:=\sup _{t \in J}\left|(\ln t)^{1-\gamma} w(t)\right|, \\
& C_{\gamma}(J)=\left\{w:(0, T] \rightarrow \mathbb{R}: t^{1-\gamma} w(t) \in C\right\}
\end{aligned}
$$


with the norm

$$
\|w\|_{C_{\gamma}}:=\sup _{t \in J}\left|t^{1-\gamma} w(t)\right|
$$

and

$$
C_{\gamma}^{1}(J)=\left\{w \in C: \frac{d w}{d t} \in C_{\gamma}\right\}
$$

with the norm

$$
\|w\|_{C_{\gamma}^{1}}:=\|w\|_{\infty}+\left\|w^{\prime}\right\|_{C_{\gamma}}
$$

In the following, we denote $\|w\|_{C_{\gamma, \mathrm{ln}}}$ by $\|w\|_{C}$.

Now, we give some results and properties of fractional calculus.

Definition 2.1 ([2-4]; Riemann-Liouville fractional integral) The left-sided mixed Riemann-Liouville integral of order $r>0$ of a function $w \in L^{1}(J)$ is defined by

$$
\left(I_{1}^{r} w\right)(t)=\frac{1}{\Gamma(r)} \int_{1}^{t}(t-s)^{r-1} w(s) d s \quad \text { for a.e. } t \in J
$$

where $\Gamma(\cdot)$ is the (Euler's) gamma function defined by

$$
\Gamma(\xi)=\int_{0}^{\infty} t^{\xi-1} e^{-t} d t ; \quad \xi>0 .
$$

Notice that for all $r, r_{1}, r_{2}>0$ and each $w \in C$, we have $I_{1}^{r} w \in C$, and

$$
\left(I_{1}^{r_{1}} I_{1}^{r_{2}} w\right)(t)=\left(I_{1}^{r_{1}+r_{2}} w\right)(t) \quad \text { for a.e. } t \in J
$$

Definition 2.2 ([2-4]; Riemann-Liouville fractional derivative) The Riemann-Liouville fractional derivative of order $r>0$ of a function $w \in L^{1}(J)$ is defined by

$$
\begin{aligned}
\left(D_{1}^{r} w\right)(t) & =\left(\frac{d^{n}}{d t^{n}} I_{1}^{n-r} w\right)(t) \\
& =\frac{1}{\Gamma(n-r)} \frac{d^{n}}{d t^{n}} \int_{1}^{t}(t-s)^{n-r-1} w(s) d s \quad \text { for a.e. } t \in J
\end{aligned}
$$

where $n=[r]+1$ and $[r]$ is the integer part of $r$.

In particular, if $r \in(0,1]$, then

$$
\begin{aligned}
\left(D_{1}^{r} w\right)(t) & =\left(\frac{d}{d t} I_{1}^{1-r} w\right)(t) \\
& =\frac{1}{\Gamma(1-r)} \frac{d}{d t} \int_{1}^{t}(t-s)^{-r} w(s) d s \quad \text { for a.e. } t \in J
\end{aligned}
$$


Let $r \in(0,1], \gamma \in[0,1)$ and $w \in C_{1-\gamma}(J)$. Then the following expression leads to the left inverse operator as follows:

$$
\left(D_{1}^{r} I_{1}^{r} w\right)(t)=w(t) \quad \text { for all } t \in(1, T]
$$

Moreover, if $I_{1}^{1-r} w \in C_{1-\gamma}^{1}(J)$, then the following composition is proved in [3]:

$$
\left(I_{1}^{r} D_{1}^{r} w\right)(t)=w(t)-\frac{\left(I_{1}^{1-r} w\right)\left(1^{+}\right)}{\Gamma(r)} t^{r-1} \quad \text { for all } t \in(1, T] .
$$

Definition 2.3 ([2-4]; Caputo fractional derivative) The Caputo fractional derivative of order $r>0$ of a function $w \in L^{1}(J)$ is defined by

$$
\begin{aligned}
\left({ }^{c} D_{1}^{r} w\right)(t) & =\left(I_{1}^{n-r} \frac{d^{n}}{d t^{n}} w\right)(t) \\
& =\frac{1}{\Gamma(n-r)} \int_{1}^{t}(t-s)^{n-r-1} \frac{d^{n}}{d s^{n}} w(s) d s \quad \text { for a.e. } t \in J .
\end{aligned}
$$

In particular, if $r \in(0,1]$, then

$$
\begin{aligned}
\left({ }^{c} D_{1}^{r} w\right)(t) & =\left(I_{1}^{1-r} \frac{d}{d t} w\right)(t) \\
& =\frac{1}{\Gamma(1-r)} \int_{1}^{t}(t-s)^{-r} \frac{d}{d s} w(s) d s \quad \text { for a.e. } t \in J
\end{aligned}
$$

Let us recall some definitions and properties of Hadamard fractional integration and differentiation. We refer to $[4,45]$ for a more detailed analysis.

Definition 2.4 ([4, 45]; Hadamard fractional integral) The Hadamard fractional integral of order $q>0$ for a function $g \in L^{1}(I, E)$ is defined as

$$
\left({ }^{H} I_{1}^{q} g\right)(x)=\frac{1}{\Gamma(q)} \int_{1}^{x}\left(\ln \frac{x}{s}\right)^{q-1} \frac{g(s)}{s} d s
$$

provided the integral exists.

Example 2.5 Let $0<q<1$. Then

$$
{ }^{H} I_{1}^{q} \ln t=\frac{1}{\Gamma(2+q)}(\ln t)^{1+q} \quad \text { for a.e. } t \in[0, e] .
$$

Set

$$
\delta=x \frac{d}{d x}, \quad q>0, \quad n=[q]+1
$$

and

$$
\operatorname{AC}_{\delta}^{n}:=\left\{u:[1, T] \rightarrow E: \delta^{n-1}[u(x)] \in \mathrm{AC}(J)\right\}
$$


Analogous to the Riemann-Liouville fractional calculus, the Hadamard fractional derivative is defined in terms of the Hadamard fractional integral in the following way.

Definition 2.6 ([4, 45]; Hadamard fractional derivative) The Hadamard fractional derivative of order $q>0$ applied to the function $w \in \mathrm{AC}_{\delta}^{n}$ is defined as

$$
\left({ }^{H} D_{1}^{q} w\right)(x)=\delta^{n}\left({ }^{H} I_{1}^{n-q} w\right)(x) .
$$

In particular, if $q \in(0,1]$, then

$$
\left({ }^{H} D_{1}^{q} w\right)(x)=\delta\left({ }^{H} I_{1}^{1-q} w\right)(x) .
$$

Example 2.7 Let $0<q<1$. Then

$$
{ }^{H} D_{1}^{q} \ln t=\frac{1}{\Gamma(2-q)}(\ln t)^{1-q} \quad \text { for a.e. } t \in[0, e] .
$$

It has been proved (see, e.g., Kilbas [46], Theorem 4.8) that in the space $L^{1}(J)$ the Hadamard fractional derivative is the left-inverse operator to the Hadamard fractional integral, i.e.,

$$
\left({ }^{H} D_{1}^{q}\right)\left({ }^{H} I_{1}^{q} w\right)(x)=w(x) .
$$

From Theorem 2.3 of [4], we have

$$
\left({ }^{H} I_{1}^{q}\right)\left({ }^{H} D_{1}^{q} w\right)(x)=w(x)-\frac{\left({ }^{H} I_{1}^{1-q} w\right)(1)}{\Gamma(q)}(\ln x)^{q-1} .
$$

Analogous to the Hadamard fractional calculus, the Caputo-Hadamard fractional derivative is defined in the following way.

Definition 2.8 (Caputo-Hadamard fractional derivative) The Caputo-Hadamard fractional derivative of order $q>0$ applied to the function $w \in \mathrm{AC}_{\delta}^{n}$ is defined as

$$
\left({ }^{H c} D_{1}^{q} w\right)(x)=\left({ }^{H} I_{1}^{n-q} \delta^{n} w\right)(x)
$$

In particular, if $q \in(0,1]$, then

$$
\left({ }^{H c} D_{1}^{q} w\right)(x)=\left({ }^{H} I_{1}^{1-q} \delta w\right)(x) .
$$

In [38], Hilfer studied applications of a generalized fractional operator having the Riemann-Liouville and the Caputo derivatives as specific cases (see also [39-41]).

Definition 2.9 (Hilfer fractional derivative) Let $\alpha \in(0,1), \beta \in[0,1], w \in L^{1}(J)$, $I_{1}^{(1-\alpha)(1-\beta)} w \in \mathrm{AC}^{1}(J)$. The Hilfer fractional derivative of order $\alpha$ and type $\beta$ of $w$ is defined as

$$
\left(D_{1}^{\alpha, \beta} w\right)(t)=\left(I_{1}^{\beta(1-\alpha)} \frac{d}{d t} I_{1}^{(1-\alpha)(1-\beta)} w\right)(t) \quad \text { for a.e. } t \in J
$$


Properties Let $\alpha \in(0,1), \beta \in[0,1], \gamma=\alpha+\beta-\alpha \beta$, and $w \in L^{1}(J)$.

1. The operator $\left(D_{1}^{\alpha, \beta} w\right)(t)$ can be written as

$$
\left(D_{1}^{\alpha, \beta} w\right)(t)=\left(I_{1}^{\beta(1-\alpha)} \frac{d}{d t} I_{1}^{1-\gamma} w\right)(t)=\left(I_{1}^{\beta(1-\alpha)} D_{1}^{\gamma} w\right)(t) \quad \text { for a.e. } t \in J .
$$

Moreover, the parameter $\gamma$ satisfies

$$
\gamma \in(0,1], \quad \gamma \geq \alpha, \quad \gamma>\beta, \quad 1-\gamma<1-\beta(1-\alpha) .
$$

2. The generalization (2) for $\beta=0$ coincides with the Riemann-Liouville derivative and for $\beta=1$ with the Caputo derivative.

$$
D_{1}^{\alpha, 0}=D_{1}^{\alpha}, \quad \text { and } \quad D_{1}^{\alpha, 1}={ }^{c} D_{1}^{\alpha}
$$

3. If $D_{1}^{\beta(1-\alpha)} w$ exists and in $L^{1}(J)$, then

$$
\left(D_{1}^{\alpha, \beta} I_{1}^{\alpha} w\right)(t)=\left(I_{1}^{\beta(1-\alpha)} D_{1}^{\beta(1-\alpha)} w\right)(t) \quad \text { for a.e. } t \in J
$$

Furthermore, if $w \in C_{\gamma}(J)$ and $I_{1}^{1-\beta(1-\alpha)} w \in C_{\gamma}^{1}(J)$, then

$$
\left(D_{1}^{\alpha, \beta} I_{1}^{\alpha} w\right)(t)=w(t) \quad \text { for a.e. } t \in J
$$

4. If $D_{1}^{\gamma} w$ exists and in $L^{1}(J)$, then

$$
\left(I_{1}^{\alpha} D_{1}^{\alpha, \beta} w\right)(t)=\left(I_{1}^{\gamma} D_{1}^{\gamma} w\right)(t)=w(t)-\frac{I_{1}^{1-\gamma}\left(1^{+}\right)}{\Gamma(\gamma)} t^{\gamma-1} \quad \text { for a.e. } t \in J .
$$

From the Hadamard fractional integral, the Hilfer-Hadamard fractional derivative (introduced for the first time in [43]) is defined in the following way.

Definition 2.10 (Hilfer-Hadamard fractional derivative) Let $\alpha \in(0,1), \beta \in[0,1], \gamma=\alpha+$ $\beta-\alpha \beta, w \in L^{1}(J)$, and ${ }^{H} I_{1}^{(1-\alpha)(1-\beta)} w \in \mathrm{AC}^{1}(J)$. The Hilfer-Hadamard fractional derivative of order $\alpha$ and type $\beta$ applied to the function $w$ is defined as

$$
\begin{aligned}
\left({ }^{H} D_{1}^{\alpha, \beta} w\right)(t) & =\left({ }^{H} I_{1}^{\beta(1-\alpha)}\left({ }^{H} D_{1}^{\gamma} w\right)\right)(t) \\
& =\left({ }^{H} I_{1}^{\beta(1-\alpha)} \delta\left({ }^{H} I_{1}^{1-\gamma} w\right)\right)(t) \quad \text { for a.e. } t \in J .
\end{aligned}
$$

This new fractional derivative (3) may be viewed as interpolating the Hadamard fractional derivative and the Caputo-Hadamard fractional derivative. Indeed, for $\beta=0$, this derivative reduces to the Hadamard fractional derivative, and when $\beta=1$, we recover the Caputo-Hadamard fractional derivative.

$$
{ }^{H} D_{1}^{\alpha, 0}={ }^{H} D_{1}^{\alpha}, \quad \text { and } \quad{ }^{H} D_{1}^{\alpha, 1}={ }^{H c} D_{1}^{\alpha} .
$$

From Theorem 21 in [44], we concluded the following lemma. 
Lemma 2.11 Let $f: I \times E \rightarrow E$ be such that $f(\cdot, u(\cdot)) \in C_{\gamma, \ln }(J)$ for any $u \in C_{\gamma, \ln }(J)$. Then problem (1) is equivalent to the problem of the solutions of the Volterra integral equation

$$
u(t)=\frac{\phi}{\Gamma(\gamma)}(\ln t)^{\gamma-1}+\left({ }^{H} I_{1}^{\alpha} f(\cdot, u(\cdot))\right)(t) .
$$

Now, we consider the Ulam stability for problem (1). Let $\epsilon>0$ and $\Phi: I \rightarrow[0, \infty)$ be a continuous function. We consider the following inequalities:

$$
\begin{aligned}
& \left|\left({ }^{H} D_{1}^{\alpha, \beta} u\right)(t)-f(t, u(t))\right| \leq \epsilon ; \quad t \in J . \\
& \left|\left({ }^{H} D_{1}^{\alpha, \beta} u\right)(t)-f(t, u(t))\right| \leq \Phi(t) ; \quad t \in J . \\
& \left|\left({ }^{H} D_{1}^{\alpha, \beta} u\right)(t)-f(t, u(t))\right| \leq \epsilon \Phi(t) ; \quad t \in J .
\end{aligned}
$$

Definition $2.12([2,31])$ Problem (1) is Ulam-Hyers stable if there exists a real number $c_{f}>0$ such that for each $\epsilon>0$ and for each solution $u \in C_{\gamma, \ln }$ of inequality (4) there exists a solution $v \in C_{\gamma, \ln }$ of (1) with

$$
|u(t)-v(t)| \leq \epsilon c_{f} ; \quad t \in J .
$$

Definition $2.13([2,31])$ Problem (1) is generalized Ulam-Hyers stable if there exists $c_{f}$ : $C([0, \infty),[0, \infty))$ with $c_{f}(0)=0$ such that for each $\epsilon>0$ and for each solution $u \in C_{\gamma, \ln }$ of inequality (4) there exists a solution $v \in C_{\gamma, \ln }$ of (1) with

$$
|u(t)-v(t)| \leq c_{f}(\epsilon) ; \quad t \in J .
$$

Definition 2.14 ([2,31]) Problem (1) is Ulam-Hyers-Rassias stable with respect to $\Phi$ if there exists a real number $c_{f, \Phi}>0$ such that for each $\epsilon>0$ and for each solution $u \in C_{\gamma, \ln }$ of inequality (6) there exists a solution $v \in C_{\gamma, \ln }$ of (1) with

$$
|u(t)-v(t)| \leq \epsilon c_{f, \Phi} \Phi(t) ; \quad t \in J .
$$

Definition 2.15 ([2, 31]) Problem (1) is generalized Ulam-Hyers-Rassias stable with respect to $\Phi$ if there exists a real number $c_{f, \Phi}>0$ such that for each solution $u \in C_{\gamma, \ln }$ of inequality (5) there exists a solution $v \in C_{\gamma, \ln }$ of (1) with

$$
|u(t)-v(t)| \leq c_{f, \Phi} \Phi(t) ; \quad t \in J .
$$

Remark 2.16 It is clear that

(i) Definition $2.12 \Rightarrow$ Definition 2.13,

(ii) Definition $2.14 \Rightarrow$ Definition 2.15,

(iii) Definition 2.14 for $\Phi(\cdot)=1 \Rightarrow$ Definition 2.12.

One can have similar remarks for inequalities (4) and (6).

In the sequel we will make use of the following fixed point theorem.

Theorem 2.17 (Schauder fixed point theorem [47]) Let E be a Banach space and $Q$ be a nonempty bounded convex and closed subset of $E$, and $N: Q \rightarrow Q$ is a compact and continuous map. Then $N$ has at least one fixed point in $Q$. 


\section{Existence of solutions}

Let us start by defining what we mean by a solution of problem (1).

Definition 3.1 By a solution of problem (1) we mean a measurable function $u \in C_{\gamma, \ln }$ that satisfies the condition $\left({ }^{H} I_{1}^{1-\gamma} u\right)\left(1^{+}\right)=\phi$ and the equation $\left({ }^{H} D_{1}^{\alpha, \beta} u\right)(t)=f(t, u(t))$ on $J$.

The following hypotheses will be used in the sequel.

( $\left.H_{1}\right)$ The function $t \mapsto f(t, u)$ is measurable on $I$ for each $u \in C_{\gamma, \ln }$, and the function $u \mapsto$ $f(t, u)$ is continuous on $C_{\gamma, \ln }$ for a.e. $t \in J$,

$\left(H_{2}\right)$ There exists a continuous function $p: I \rightarrow[0, \infty)$ such that

$$
|f(t, u)| \leq \frac{p(t)}{1+|u|}|u| \quad \text { for a.e. } t \in J \text { and each } u \in \mathbb{R} \text {. }
$$

Set

$$
p^{*}=\sup _{t \in J} p(t)
$$

Now, we shall prove the following theorem concerning the existence of solutions of problem (1).

Theorem 3.2 Assume that hypotheses $\left(H_{1}\right)$ and $\left(H_{2}\right)$ hold. Then problem (1) has at least one solution defined on $J$.

Proof Consider the operator $N: C_{\gamma, \ln } \rightarrow C_{\gamma, \ln }$ defined by

$$
(N u)(t)=\frac{\phi}{\Gamma(\gamma)}(\ln t)^{\gamma-1}+\int_{1}^{t}\left(\ln \frac{t}{s}\right)^{\alpha-1} \frac{f(s, u(s))}{s \Gamma(\alpha)} d s .
$$

Clearly, the fixed points of the operator $N$ are solution of problem (1).

For any $u \in C_{\gamma, \text { ln }}$ and each $t \in J$, we have

$$
\begin{aligned}
\left|(\ln t)^{1-\gamma}(N u)(t)\right| & \leq \frac{|\phi|}{\Gamma(\gamma)}+\frac{(\ln t)^{1-\gamma}}{\Gamma(\alpha)} \int_{1}^{t}\left(\ln \frac{t}{s}\right)^{\alpha-1}|f(s, u(s))| \frac{d s}{s} \\
& \leq \frac{|\phi|}{\Gamma(\gamma)}+\frac{(\ln t)^{1-\gamma}}{\Gamma(\alpha)} \int_{1}^{t}\left(\ln \frac{t}{s}\right)^{\alpha-1} p(s) \frac{d s}{s} \\
& \leq \frac{|\phi|}{\Gamma(\gamma)}+\frac{p^{*}(\ln T)^{1-\gamma}}{\Gamma(\alpha)} \int_{1}^{t}\left(\ln \frac{t}{s}\right)^{\alpha-1} \frac{d s}{s} \\
& \leq \frac{|\phi|}{\Gamma(\gamma)}+\frac{p^{*}(\ln T)^{1-\gamma+\alpha}}{\Gamma(1+\alpha)} .
\end{aligned}
$$

Thus

$$
\|N(u)\|_{C} \leq \frac{|\phi|}{\Gamma(\gamma)}+\frac{p^{*}(\ln T)^{1-\gamma+\alpha}}{\Gamma(1+\alpha)}:=R .
$$

This proves that $N$ transforms the ball $B_{R}:=B(0, R)=\left\{w \in C_{\gamma, \mathrm{ln}}:\|w\|_{C} \leq R\right\}$ into itself. We shall show that the operator $N: B_{R} \rightarrow B_{R}$ satisfies all the assumptions of Theorem 2.17. The proof will be given in several steps. 
Step 1. $N: B_{R} \rightarrow B_{R}$ is continuous.

Let $\left\{u_{n}\right\}_{n \in \mathbb{N}}$ be a sequence such that $u_{n} \rightarrow u$ in $B_{R}$. Then, for each $t \in J$, we have

$$
\begin{aligned}
& \left|(\ln t)^{1-\gamma}\left(N u_{n}\right)(t)-(\ln t)^{1-\gamma}(N u)(t)\right| \\
& \quad \leq \frac{(\ln t)^{1-\gamma}}{\Gamma(\alpha)} \int_{1}^{t}\left(\ln \frac{t}{s}\right)^{\alpha-1}\left|f\left(s, u_{n}(s)\right)-f(s, u(s))\right| \frac{d s}{s} .
\end{aligned}
$$

Since $u_{n} \rightarrow u$ as $n \rightarrow \infty$ and $f$ is continuous, by the Lebesgue dominated convergence theorem, equation (9) implies

$$
\left\|N\left(u_{n}\right)-N(u)\right\|_{C} \rightarrow 0 \quad \text { as } n \rightarrow \infty .
$$

Step 2. $N\left(B_{R}\right)$ is uniformly bounded.

This is clear since $N\left(B_{R}\right) \subset B_{R}$ and $B_{R}$ is bounded.

Step 3. $N\left(B_{R}\right)$ is equicontinuous.

Let $t_{1}, t_{2} \in J, t_{1}<t_{2}$ and let $u \in B_{R}$. Thus, we have

$$
\begin{aligned}
& \left|\left(\ln t_{2}\right)^{1-\gamma}(N u)\left(t_{2}\right)-\left(\ln t_{1}\right)^{1-\gamma}(N u)\left(t_{1}\right)\right| \\
& \leq\left|\left(\ln t_{2}\right)^{1-\gamma} \int_{1}^{t_{2}}\left(\ln \frac{t_{2}}{s}\right)^{\alpha-1} \frac{f(s, u(s))}{s \Gamma(\alpha)} d s-\left(\ln t_{1}\right)^{1-\gamma} \int_{1}^{t_{1}}\left(\ln \frac{t_{1}}{s}\right)^{\alpha-1} \frac{f(s, u(s))}{s \Gamma(\alpha)} d s\right| \\
& \leq\left(\ln t_{2}\right)^{1-\gamma} \int_{t_{1}}^{t_{2}}\left(\ln \frac{t_{2}}{s}\right)^{\alpha-1} \frac{|f(s, u(s))|}{s \Gamma(\alpha)} d s \\
& \quad+\int_{1}^{t_{1}}\left|\left(\ln t_{2}\right)^{1-\gamma}\left(\ln \frac{t_{2}}{s}\right)^{\alpha-1}-\left(\ln t_{1}\right)^{1-\gamma}\left(\ln \frac{t_{1}}{s}\right)^{\alpha-1}\right| \frac{|f(s, u(s))|}{s \Gamma(\alpha)} d s \\
& \leq\left(\ln t_{2}\right)^{1-\gamma} \int_{t_{1}}^{t_{2}}\left(\ln \frac{t_{2}}{s}\right)^{\alpha-1} \frac{p(s)}{s \Gamma(\alpha)} d s \\
& \quad+\int_{1}^{t_{1}}\left|\left(\ln t_{2}\right)^{1-\gamma}\left(\ln \frac{t_{2}}{s}\right)^{\alpha-1}-\left(\ln t_{1}\right)^{1-\gamma}\left(\ln \frac{t_{1}}{s}\right)^{\alpha-1}\right| \frac{p(s)}{s \Gamma(\alpha)} d s .
\end{aligned}
$$

Hence, we get

$$
\begin{aligned}
& \left|\left(\ln t_{2}\right)^{1-\gamma}(N u)\left(t_{2}\right)-\left(\ln t_{1}\right)^{1-\gamma}(N u)\left(t_{1}\right)\right| \\
& \leq \frac{p_{*}(\ln T)^{1-\gamma+\alpha}}{\Gamma(1+\alpha)}\left(\ln \frac{t_{2}}{t_{1}}\right)^{\alpha} \\
& \quad+\frac{p_{*}}{\Gamma(\alpha)} \int_{1}^{t_{1}}\left|\left(\ln t_{2}\right)^{1-\gamma}\left(\ln \frac{t_{2}}{s}\right)^{\alpha-1}-\left(\ln t_{1}\right)^{1-\gamma}\left(\ln \frac{t_{1}}{s}\right)^{\alpha-1}\right| d s .
\end{aligned}
$$

As $t_{1} \longrightarrow t_{2}$, the right-hand side of the above inequality tends to zero.

As a consequence of Steps 1 to 3 together with the Arzelá-Ascoli theorem, we can conclude that $N$ is continuous and compact. From an application of Schauder's theorem (Theorem 2.17), we deduce that $N$ has at least a fixed point $u$ which is a solution of problem (1).

\section{Ulam-Hyers-Rassias stability}

Now, we are concerned with the generalized Ulam-Hyers-Rassias stability of our problem (1). 
Theorem 4.1 Assume that hypotheses $\left(H_{1}\right),\left(H_{2}\right)$ and the following hypotheses hold.

$\left(H_{3}\right)$ There exists $\lambda_{\Phi}>0$ such that for each $t \in J$, we have

$$
\left({ }^{H} I_{1}^{\alpha} \Phi\right)(t) \leq \lambda_{\Phi} \Phi(t)
$$

$\left(H_{4}\right)$ There exists $q \in C(J,[0, \infty))$ such that for each $t \in J$, we have

$$
p(t) \leq q(t) \Phi(t)
$$

Then problem (1) is generalized Ulam-Hyers-Rassias stable.

Proof Consider the operator $N: C_{\gamma, \ln } \rightarrow C_{\gamma, \ln }$ defined in (7). Let $u$ be a solution of inequality (5), and let us assume that $v$ is a solution of problem (1). Thus, we have

$$
v(t)=\frac{\phi}{\Gamma(\gamma)}(\ln t)^{\gamma-1}+\int_{1}^{t}\left(\ln \frac{t}{s}\right)^{\alpha-1} \frac{f(s, v(s))}{s \Gamma(\alpha)} d s .
$$

From inequality (5), for each $t \in J$, we have

$$
\left|u(t)-\frac{\phi}{\Gamma(\gamma)}(\ln t)^{\gamma-1}-\int_{1}^{t}\left(\ln \frac{t}{s}\right)^{\alpha-1} \frac{f(s, u(s))}{s \Gamma(\alpha)} d s\right| \leq\left({ }^{H} I_{1}^{\alpha} \Phi\right)(t) .
$$

Set

$$
q^{*}=\sup _{t \in J} q(t)
$$

From hypotheses $\left(H_{3}\right)$ and $\left(H_{4}\right)$, for each $t \in J$, we get

$$
\begin{aligned}
|u(t)-v(t)| \leq & \left|u(t)-\frac{\phi}{\Gamma(\gamma)}(\ln t)^{\gamma-1}-\int_{1}^{t}\left(\ln \frac{t}{s}\right)^{\alpha-1} \frac{f(s, u(s))}{s \Gamma(\alpha)} d s\right| \\
& +\int_{1}^{t}\left(\ln \frac{t}{s}\right)^{\alpha-1} \frac{|f(s, u(s))-f(s, v(s))|}{s \Gamma(\alpha)} d s \\
\leq & \left({ }^{H} I_{1}^{\alpha} \Phi\right)(t)+\int_{1}^{t}\left(\ln \frac{t}{s}\right)^{\alpha-1} \frac{2 q^{*} \Phi(s)}{s \Gamma(\alpha)} d s \\
\leq & \lambda_{\phi} \Phi(t)+2 q^{*}\left({ }^{H} I_{1}^{\alpha} \Phi\right)(t) \\
\leq & {\left[1+2 q^{*}\right] \lambda_{\phi} \Phi(t) } \\
:= & c_{f, \Phi} \Phi(t) .
\end{aligned}
$$

Hence, problem (1) is generalized Ulam-Hyers-Rassias stable.

In the sequel, we will use the following theorem.

Theorem 4.2 Let $(\Omega, d)$ be a generalized complete metric space and $\Theta: \Omega \rightarrow \Omega$ be a strictly contractive operator with a Lipschitz constant $L<1$. If there exists a nonnegative integer $k$ such that $d\left(\Theta^{k+1} x, \Theta^{k} x\right)<\infty$ for some $x \in \Omega$, then the following propositions hold true: 
(A) The sequence $\left(\Theta^{k} x\right)_{n \in N}$ converges to a fixed point $x^{*}$ of $\Theta$;

(B) $x^{*}$ is the unique fixed point of $\Theta$ in $\Omega^{*}=\left\{y \in \Omega \mid d\left(\Theta^{k} x, y\right)<\infty\right\}$;

(C) If $y \in \Omega^{*}$, then $d\left(y, x^{*}\right) \leq \frac{1}{1-L} d(y, \Theta x)$.

Let $X=X(I, \mathbb{R})$ be the metric space, with the metric

$$
d(u, v)=\sup _{t \in J} \frac{\|u(t)-v(t)\|_{C}}{\Phi(t)} .
$$

Theorem 4.3 Assume that $\left(H_{3}\right)$ and the following hypothesis hold.

$\left(H_{5}\right)$ There exists $\varphi \in C(J,[0, \infty))$ such that for each $t \in J$ and all $u, v \in \mathbb{R}$, we have

$$
|f(t, u)-f(t, u)| \leq(\ln t)^{1-\gamma} \varphi(t) \Phi(t)|u-v|
$$

If

$$
L:=(\ln T)^{1-\gamma} \varphi^{*} \lambda_{\phi}<1
$$

where $\varphi^{*}=\sup _{t \in J} \varphi(t)$, then there exists a unique solution $u_{0}$ of problem (1), and problem (1) is generalized Ulam-Hyers-Rassias stable. Furthermore, we have

$$
\left|u(t)-u_{0}(t)\right| \leq \frac{\Phi(t)}{1-L}
$$

Proof Let $N: C_{\gamma, \ln } \rightarrow C_{\gamma, \ln }$ be the operator defined in (7). Applying Theorem 4.2, we have

$$
\begin{aligned}
|(N u)(t)-(N v)(t)| & \leq \int_{1}^{t}\left(\ln \frac{t}{s}\right)^{\alpha-1} \frac{|f(s, u(s))-f(s, v(s))|}{s \Gamma(\alpha)} d s \\
& \leq \int_{1}^{t}\left(\ln \frac{t}{s}\right)^{\alpha-1} \frac{\varphi(s) \Phi(s)\left|(\ln s)^{1-\gamma} u(s)-(\ln s)^{1-\gamma} v(s)\right|}{s \Gamma(\alpha)} d s \\
& \leq \int_{1}^{t}\left(\ln \frac{t}{s}\right)^{\alpha-1} \frac{\varphi^{*} \Phi(s)\|u-v\|_{C}}{s \Gamma(\alpha)} d s \\
& \leq \varphi^{*}\left({ }^{H} I_{1}^{\alpha} \Phi\right)(t)\|u-v\|_{C} \\
& \leq \varphi^{*} \lambda_{\phi} \Phi(t)\|u-v\|_{C} .
\end{aligned}
$$

Thus

$$
\left|(\ln t)^{1-\gamma}(N u)(t)-(\ln t)^{1-\gamma}(N v)(t)\right| \leq(\ln T)^{1-\gamma} \varphi^{*} \lambda_{\phi} \Phi(t)\|u-v\|_{C} .
$$

Hence, we get

$$
d(N(u), N(v))=\sup _{t \in J} \frac{\|(N u)(t)-(N v)(t)\|_{C}}{\Phi(t)} \leq L\|u-v\|_{C},
$$

from which we conclude the theorem. 


\section{An example}

As an application of our results, we consider the following problem of Hilfer-Hadamard fractional differential equation of the form

$$
\left\{\begin{array}{l}
\left({ }^{H} D_{1}^{\frac{1}{2}, \frac{1}{2}} u\right)(t)=f(t, u(t)) ; \quad t \in[1, e], \\
\left.\left({ }^{H} I_{1}^{\frac{1}{4}} u\right)(t)\right|_{t=1}=0
\end{array}\right.
$$

where

$$
\left\{\begin{array}{l}
f(t, u)=\frac{(t-1)^{\frac{-1}{4}} \sin (t-1)}{64(1+\sqrt{t-1})(1+|u|)} ; \quad t \in(1, e], u \in \mathbb{R}, \\
f(1, u)=0 ; \quad u \in \mathbb{R} .
\end{array}\right.
$$

Clearly, the function $f$ is continuous.

Hypothesis $\left(H_{2}\right)$ is satisfied with

$$
\left\{\begin{array}{l}
p(t)=\frac{(t-1)^{\frac{-1}{4}}|\sin (t-1)|}{64(1+\sqrt{t-1})} ; \quad t \in(1, e] \\
p(1)=0
\end{array}\right.
$$

Hence, Theorem 3.2 implies that problem (11) has at least one solution defined on $[1, e]$. Also, hypothesis $\left(H_{3}\right)$ is satisfied with

$$
\Phi(t)=e^{3}, \quad \text { and } \quad \lambda_{\Phi}=\frac{2}{\sqrt{\pi}} .
$$

Indeed, for each $t \in[1, e]$, we get

$$
\begin{aligned}
\left({ }^{H} I_{1}^{\alpha} \Phi\right)(t) & \leq \frac{2 e^{3}}{\sqrt{\pi}} \\
& =\lambda_{\Phi} \Phi(t) .
\end{aligned}
$$

Consequently, Theorem 4.1 implies that problem (11) is generalized Ulam-Hyers-Rassias stable.

\section{Acknowledgements}

The authors would like to thank all the anonymous reviewers and the editors for their helpful advice and hard work. The work was supported by the National Natural Science Foundation of China (No. 11671339).

\section{Competing interests}

The authors declare that they have no competing interests.

\section{Authors' contributions}

$\mathrm{SA}, \mathrm{MB}$, and JEL contributed to Sections 1, 2,3, and 4. AA and YZ contributed to Sections 1 and 5 .

\section{Author details}

'Laboratory of Mathematics, University of Saïda, P.O. Box 138, Saïda, 20000, Algeria. ${ }^{2}$ Laboratory of Mathematics, University of Sidi Bel-Abbes, P.O. Box 89, Sidi Bel-Abbès, 22000, Algeria. ${ }^{3}$ Nonlinear Analysis and Applied Mathematics (NAAM) Research Group, Faculty of Science, King Abdulaziz University, P.O. Box 80203, Jeddah, 21589, Saudi Arabia. ${ }^{4}$ Faculty of Mathematics and Computational Science, Xiangtan University, Hunan, 411105, P.R. China.

\section{Publisher's Note}

Springer Nature remains neutral with regard to jurisdictional claims in published maps and institutional affiliations. 


\section{References}

1. Abbas, S, Benchohra, M: Advanced Functional Evolution Equations and Inclusions. Developments in Mathematics, vol. 39. Springer, Cham (2015)

2. Abbas, S, Benchohra, M, N'Guérékata, GM: Topics in Fractional Differential Equations. Springer, New York (2012)

3. Samko, SG, Kilbas, AA, Marichev, Ol: Fractional Integrals and Derivatives. Theory and Applications. Gordon \& Breach, Amsterdam (1987). Engl. Trans. from the Russian

4. Kilbas, AA, Srivastava, HM, Trujillo, JJ: Theory and Applications of Fractional Differential Equations. Elsevier, Amsterdam (2006)

5. Zhou, Y: Basic Theory of Fractional Differential Equations. World Scientific, Singapore (2014)

6. Abbas, S, Benchohra, M: Uniqueness and Ulam stabilities results for partial fractional differential equations with not instantaneous impulses. Appl. Math. Comput. 257, 190-198 (2015)

7. Abbas, S, Benchohra, M: Existence and Ulam stability for impulsive discontinuous fractional differential inclusions in Banach algebras. Mediterr. J. Math. 12(4), 1245-1264 (2015)

8. Abbas, S, Benchohra, M, Petrusel, A: Ulam stabilities for the Darboux problem for partial fractional differential inclusions via Picard operators. Electron. J. Qual. Theory Differ. Equ. 2014, 51 (2014)

9. Abbas, S, Benchohra, M, Sivasundaram, S: Ulam stability for partial fractional differential inclusions with multiple delay and impulses via Picard operators. Nonlinear Stud. 20(4), 623-641 (2013)

10. Ahmad, B, Alsaedi, A: Existence and uniqueness of solutions for coupled systems of higher-order nonlinear fractional differential equations. Fixed Point Theory Appl. 2010, 364560 (2010)

11. Ahmad, B, Nieto, JJ, Alsaedi, A, et al.: A study of nonlinear Langevin equation involving two fractional orders in different intervals. Nonlinear Anal., Real World Appl. 13, 599-606 (2012)

12. Benchohra, M, Henderson, J, Ntouyas, SK, Ouahab, A: Existence results for functional differential equations of fractional order. J. Math. Anal. Appl. 338, 1340-1350 (2008)

13. Benchohra, M, Souid, MS: Integrable solutions for implicit fractional order differential equations. Transylv. J. Math. Mech. 6, 101-107 (2014)

14. Benchohra, M, Souid, MS: Integrable solutions for implicit fractional order functional differential equations with infinite delay. Arch. Math. 51, 13-22 (2015)

15. Wang, J-R, Feckan, M, Zhou, Y: A survey on impulsive fractional differential equations. Fract. Calc. Appl. Anal. 19 806-831 (2016)

16. Wang, J-R, Feckan, M, Zhou, Y: Center stable manifold for planar fractional damped equations. Appl. Math. Comput. 296, 257-269 (2017)

17. Zhou, Y, Zhang, L, Shen, XH: Existence of mild solutions for fractional evolution equations. J. Integral Equ. Appl. 25, 557-586 (2013)

18. Zhou, Y, Peng, L: On the time-fractional Navier-Stokes equations. Comput. Math. Appl. 73(6), 874-891 (2017)

19. Zhou, Y, Peng, L: Weak solution of the time-fractional Navier-Stokes equations and optimal control. Comput. Math. Appl. 73(6), 1016-1027 (2017)

20. Zhou, Y, Zhang, L: Existence and multiplicity results of homoclinic solutions for fractional Hamiltonian systems. Comput. Math. Appl. 73(6), 1325-1345 (2017)

21. Zhou, Y, Ahmad, B, Alsaedi, A: Existence of nonoscillatory solutions for fractional neutral differential equations. Appl. Math. Lett. 72, 70-74 (2017)

22. Zhou, Y, Vijayakumar, V, Murugesu, R: Controllability for fractional evolution inclusions without compactness. Evol. Equ. Control Theory 4, 507-524 (2015)

23. Ulam, SM: A Collection of Mathematical Problems. Interscience, New York (1968)

24. Hyers, DH: On the stability of the linear functional equation. Proc. Natl. Acad. Sci. 27, 222-224 (1941)

25. Rassias, TM: On the stability of linear mappings in Banach spaces. Proc. Am. Math. Soc. 72, $297-300$ (1978)

26. Jung, S-M: Hyers-Ulam-Rassias Stability of Functional Equations in Mathematical Analysis. Hadronic Press, Palm Harbor (2001)

27. Abbas, S, Albarakati, W, Benchohra, M, N'Guérékata, GM: Existence and Ulam stabilities for Hadamard fractional integral equations in Fréchet spaces. J. Fract. Calc. Appl. 7(2), 1-12 (2016)

28. Abbas, S, Albarakati, WA, Benchohra, M, Sivasundaram, S: Dynamics and stability of Fredholm type fractional order Hadamard integral equations. Nonlinear Stud. 22(4), 673-686 (2015)

29. Abbas, S, Benchohra, M: Existence and Ulam stability results for quadratic integral equations. Libertas Math. 35(2) 83-93 (2015)

30. Petru, TP, Petrusel, A, Yao, J-C: Ulam-Hyers stability for operatorial equations and inclusions via nonself operators. Taiwan. J. Math. 15, 2169-2193 (2011)

31. Rus, IA: Ulam stability of ordinary differential equations. Stud. Univ. Babeş-Bolyai, Math. LIV(4), 125-133 (2009)

32. Rus, IA: Remarks on Ulam stability of the operatorial equations. Fixed Point Theory 10, 305-320 (2009)

33. Wang, J-R, Li, X: A uniform method to Ulam-Hyers stability for some linear fractional equations. Mediterr. J. Math. 13, 625-635 (2016)

34. Wang, J-R, Lin, Z, Zhou, Y: On the stability of new impulsive ordinary differential equations. Topol. Methods Nonlinear Anal. 46, 303-314 (2015)

35. Jung, S-M: Hyers-Ulam-Rassias Stability of Functional Equations in Nonlinear Analysis. Springer, New York (2011)

36. Furati, KM, Kassim, MD: Non-existence of global solutions for a differential equation involving Hilfer fractional derivative. Electron. J. Differ. Equ. 2013, 235 (2013)

37. Furati, KM, Kassim, MD, Tatar, N-E: Existence and uniqueness for a problem involving Hilfer fractional derivative. Comput. Math. Appl. 64, 1616-1626 (2012)

38. Hilfer, R: Applications of Fractional Calculus in Physics. World Scientific, Singapore (2000)

39. Hilfer, R: Threefold introduction to fractional derivatives. In: Anomalous Transport: Foundations and Applications, pp. 17-73 (2008)

40. Kamocki, R, Obczńnski, C: On fractional Cauchy-type problems containing Hilfer's derivative. Electron. J. Qual. Theory Differ. Equ. 2016, 50 (2016)

41. Tomovski, Ž, Hilfer, R, Srivastava, HM: Fractional and operational calculus with generalized fractional derivative operators and Mittag-Leffler type functions. Integral Transforms Spec. Funct. 21(11), 797-814 (2010) 
42. Wang, J-R, Zhang, Y: Nonlocal initial value problems for differential equations with Hilfer fractional derivative. Appl. Math. Comput. 266, 850-859 (2015)

43. Qassim, MD, Furati, KM, Tatar, N-E: On a differential equation involving Hilfer-Hadamard fractional derivative. Abstr. Appl. Anal. 2012, Article ID 391062 (2012)

44. Qassim, MD, Tatar, N-E: Well-posedness and stability for a differential problem with Hilfer-Hadamard fractional derivative. Abstr. Appl. Anal. 2013, Article ID 605029 (2013)

45. Hadamard, J: Essai sur l'étude des fonctions données par leur développment de Taylor. J. Math. Pures Appl. 4(8), 101-186 (1892)

46. Kilbas, AA: Hadamard-type fractional calculus. J. Korean Math. Soc. 38(6), 1191-1204 (2001)

47. Granas, A, Dugundji, J: Fixed Point Theory. Springer, New York (2003)

Submit your manuscript to a SpringerOpen ${ }^{\circ}$ journal and benefit from:

- Convenient online submission

- Rigorous peer review

- Open access: articles freely available online

- High visibility within the field

- Retaining the copyright to your article

Submit your next manuscript at $\gg$ springeropen.com 